Bull. Korean Math. Soc. 50 (2013), No. 2, pp. 451-458

http://dx.doi.org/10.4134/BKMS.2013.50.2.451

\title{
HYBRID MEAN VALUE OF THE GENERALIZED KLOOSTERMAN SUMS AND DIRICHLET CHARACTER OF POLYNOMIALS
}

\author{
WANG JiNGZHE
}

\begin{abstract}
The main purpose of this paper is using the analytic methods and the properties of Gauss sums to study the computational problem of one kind mean value of the generalized Kloosterman sums and Dirichlet character of polynomials, and give an interesting asymptotic formula for it.
\end{abstract}

\section{Introduction}

Let $q \geq 3$ be an integer, and let $\chi$ be a Dirichlet character mod $q$. It is a very important and difficult problem in analytic number theory to give a sharper upper bound estimate for the character sums of the polynomials

$$
\sum_{a=N+1}^{N+M} \chi(f(a)),
$$

where $M$ and $N$ are any positive integers, and $f(x)$ is a polynomial. For some special cases, such as $f(x)=x$, Pólya and Vinogradov's classical work (see Theorem 8.21 of [1]) proved that for any non-principal character $\chi \bmod q$, we have

$$
\sum_{a=N+1}^{N+M} \chi(a) \ll q^{\frac{1}{2}} \ln q .
$$

If $q=p$ is an odd prime, then Weil (see [3]) obtained following important conclusion:

Received October 9, 2011; Revised December 15, 2011.

2010 Mathematics Subject Classification. 11M20.

Key words and phrases. hybrid mean value, generalized Kloosterman sums, Dirichlet character of polynomials, asymptotic formula.

This work is supported by the N.S.F. (11071194) of P. R. China and the G.I.C.F. (YZZ12062) of NWU. 
Let $\chi$ be a $q$ th-order character $\bmod p$. If $f(x)$ is not a perfect $q$ th power $\bmod p$, then we have the estimate

$$
\sum_{x=N+1}^{N+M} \chi(f(x)) \ll p^{\frac{1}{2}} \ln p
$$

where $A \ll B$ denotes $|A|<c B$ for some constant $c$, which in this case depends only on the degree of $f(x)$. Some related results can also be found in [2], [3], [4], [5] and [6]. The main term $p^{\frac{1}{2}}$ in (1) is the best possible. In fact, W. Zhang and Y. Yi [4] found some polynomials $f(x)=(x-r)^{m}(x-s)^{n}$ such that

$$
\left|\sum_{a=1}^{q} \chi\left((a-r)^{m}(a-s)^{n}\right)\right|=\sqrt{q}
$$

where $(r-s, q)=1, m, n$ and $\chi$ also satisfying some special conditions.

In this paper, we consider the hybrid mean value of the generalized Kloosterman sums and the Dirichlet character of polynomials

$$
\sum_{m=1}^{q},\left|\sum_{a=1}^{q} \prime \lambda(a) e\left(\frac{m a+\bar{a}}{q}\right)\right|^{2} \cdot\left|\sum_{b=1}^{q}{ }^{\prime} \chi(m b+\bar{b})\right|^{2},
$$

where $q \geq 3$ is a positive integer, $\lambda$ and $\chi$ are two Dirichlet characters $\bmod q$, and $a \cdot \bar{a} \equiv 1 \bmod q$.

For some special integer $q$, such as $q=p$, an odd prime, using the classical analytic number theory methods one can get the estimates

$$
\left|\sum_{b=1}^{p-1} \lambda(b) e\left(\frac{m a+\bar{a}}{p}\right)\right| \leq \sqrt{p} \cdot \ln p
$$

and

$$
\left|\sum_{a=1}^{p-1} \chi(m a+\bar{a})\right| \leq 2 \sqrt{p}
$$

But for the hybrid mean value (2) of the generalized Kloosterman sums and Dirichlet character of polynomials, we do not know whether there exists a mean value formula for it?

In this paper, we shall use the analytic method and the properties of Gauss sums to study this problem, and give a sharp asymptotic formula for (2) with $q=p$, an odd prime. That is, we shall prove the following conclusion:

Theorem. Let $p$ be an odd prime. Then for any non-principal even character $\chi \bmod p$ and any character $\lambda \bmod p$ with $\lambda \neq\left(\frac{*}{p}\right)$, we have the asymptotic formula

$$
\sum_{m=1}^{p-1}\left|\sum_{a=1}^{p-1} \lambda(a) e\left(\frac{m a+\bar{a}}{p}\right)\right|^{2} \cdot\left|\sum_{b=1}^{p-1} \chi(m b+\bar{b})\right|^{2}=2 p^{3}+O\left(p^{2}\right)
$$


where $\left(\frac{*}{p}\right)$ denotes the Legendre's symbol.

From this Theorem we may immediately deduce the following:

Corollary. Let $p>3$ be a prime. Then for any non-principal even character $\chi \bmod p$, we have the asymptotic formula

$$
\sum_{m=1}^{p-1}\left|\sum_{b=1}^{p-1} e\left(\frac{m b+\bar{b}}{p}\right)\right|^{2} \cdot\left|\sum_{a=1}^{p-1} \chi(m a+\bar{a})\right|^{2}=2 p^{3}+O\left(p^{2}\right) .
$$

In the process of proving our theorem, we found that the error term is big as same as the main term, if $\lambda=\left(\frac{*}{p}\right)$. So in this case, we can not give an effective asymptotic formula for (2) with $q=p$, an odd prime. Because of this reason, we proposed the following two open problems:

A. If $\lambda=\left(\frac{*}{p}\right)$, how about the asymptotic properties in our theorem?

B. For general $\bmod q$, whether there exists an asymptotic formula for (2)?

\section{Several lemmas}

To complete the proof of our theorem, we need the following several lemmas.

Lemma 1. Let $p$ be an odd prime, $\chi$ be any non-principal even character $\bmod p$. Then for any integer $m$ with $(m, p)=1$, we have the identity

$$
\sum_{a=1}^{p-1} \chi(m a+\bar{a})=\frac{\chi_{1}(m) \tau^{2}\left(\bar{\chi}_{1}\right)}{\tau(\bar{\chi})}\left(1+\left(\frac{m}{p}\right)\left(\frac{\tau\left(\bar{\chi}_{1} \chi_{2}\right)}{\tau\left(\bar{\chi}_{1}\right)}\right)^{2}\right)
$$

where $\tau(\chi)=\sum_{a=1}^{p-1} \chi(a) e\left(\frac{a}{p}\right)$ denotes the classical Gauss sums, $\chi=\chi_{1}^{2}$, $\left(\frac{*}{p}\right)=\chi_{2}$ denotes the Legendre's symbol.

Proof. Since $\chi$ is a non-principal even character $\bmod p$, so $\chi(-1)=1$. Therefore, there exists one and only one primitive character $\chi_{1} \bmod p$ such that $\chi=\chi_{1}^{2}$. Then from the properties of Gauss sums we have

$$
\begin{aligned}
& \sum_{a=1}^{p-1} \chi(m a+\bar{a}) \\
= & \frac{1}{\tau(\bar{\chi})} \sum_{a=1}^{p-1} \sum_{b=1}^{p-1} \bar{\chi}(b) e\left(\frac{b(m a+\bar{a})}{p}\right) \\
= & \frac{1}{\tau(\bar{\chi})} \sum_{a=1}^{p-1} \bar{\chi}(a) \sum_{b=1}^{p-1} \bar{\chi}(b) e\left(\frac{b\left(m a^{2}+1\right)}{p}\right) \\
= & \frac{1}{\tau(\bar{\chi})} \sum_{b=1}^{p-1} \bar{\chi}(b) e\left(\frac{b}{p}\right) \sum_{a=1}^{p-1} \bar{\chi}(a) e\left(\frac{b m a^{2}}{p}\right)
\end{aligned}
$$




$$
\begin{aligned}
& =\frac{1}{\tau(\bar{\chi})} \sum_{b=1}^{p-1} \bar{\chi}(b) e\left(\frac{b}{p}\right) \sum_{a=1}^{p-1} \bar{\chi}_{1}\left(a^{2}\right) e\left(\frac{b m a^{2}}{p}\right) \\
& =\frac{1}{\tau(\bar{\chi})} \sum_{b=1}^{p-1} \bar{\chi}(b) e\left(\frac{b}{p}\right) \sum_{a=1}^{p-1}\left(1+\chi_{2}(a)\right) \bar{\chi}_{1}(a) e\left(\frac{b m a}{p}\right) \\
& =\frac{1}{\tau(\bar{\chi})} \sum_{b=1}^{p-1} \bar{\chi}(b) e\left(\frac{b}{p}\right)\left(\sum_{a=1}^{p-1} \bar{\chi}_{1}(a) e\left(\frac{b m a}{p}\right)+\sum_{a=1}^{p-1} \chi_{2}(a) \bar{\chi}_{1}(a) e\left(\frac{b m a}{p}\right)\right) \\
& =\frac{1}{\tau(\bar{\chi})} \sum_{b=1}^{p-1} \bar{\chi}(b) e\left(\frac{b}{p}\right)\left(\chi_{1}(b m) \tau\left(\bar{\chi}_{1}\right)+\chi_{1}(b m) \chi_{2}(b m) \tau\left(\bar{\chi}_{1} \chi_{2}\right)\right) \\
& =\frac{\chi_{1}(m) \tau\left(\bar{\chi}_{1}\right)}{\tau(\bar{\chi})}\left(\sum_{b=1}^{p-1} \bar{\chi}_{1}(b) e\left(\frac{b}{p}\right)+\chi_{2}(m) \frac{\tau\left(\bar{\chi}_{1} \chi_{2}\right)}{\tau\left(\bar{\chi}_{1}\right)} \sum_{b=1}^{p-1} \bar{\chi}_{1}(b) \chi_{2}(b) e\left(\frac{b}{p}\right)\right) \\
& =\frac{\chi_{1}(m) \tau^{2}\left(\bar{\chi}_{1}\right)}{\tau(\bar{\chi})}\left(1+\left(\frac{m}{p}\right)\left(\frac{\tau\left(\bar{\chi}_{1} \chi_{2}\right)}{\tau\left(\bar{\chi}_{1}\right)}\right)^{2}\right),
\end{aligned}
$$

where we have used the identities $\chi=\chi_{1}^{2}$ and

$$
\sum_{a=1}^{p-1} \bar{\chi}_{1}(a) e\left(\frac{m a}{p}\right)=\chi_{1}(m) \tau\left(\bar{\chi}_{1}\right) .
$$

This proves Lemma 1.

Lemma 2. Let $p$ be an odd prime, $\chi$ be any non-principal even character $\bmod p$ and $\chi=\chi_{1}^{2}$. Then we have the identity

$$
\left(\frac{\tau\left(\bar{\chi}_{1} \chi_{2}\right)}{\tau\left(\bar{\chi}_{1}\right)}\right)^{2}+\overline{\left(\frac{\tau\left(\bar{\chi}_{1} \chi_{2}\right)}{\tau\left(\bar{\chi}_{1}\right)}\right)^{2}}=\frac{(-1)^{\frac{p-1}{2}}}{p} \cdot \sum_{a=1}^{p-1} \chi(a) \sum_{b=1}^{p-1}\left(\frac{b(b-1)\left(1-a^{2} b\right)}{p}\right),
$$

where $\left(\frac{*}{p}\right)=\chi_{2}$ denotes the Legendre's symbol.

Proof. From the properties of the classical Gauss sums we know that

$$
\tau\left(\bar{\chi}_{1}\right) \overline{\tau\left(\bar{\chi}_{1}\right)}=p,
$$

using this properties and note that the identities $\chi=\chi_{1}^{2},\left(\frac{a}{p}\right)=\left(\frac{\bar{a}}{p}\right)$ and

$$
\left(\sum_{b=1}^{p-1} \chi_{2}(b) e\left(\frac{b}{p}\right)\right)^{2}=(-1)^{\frac{p-1}{2}} \cdot p
$$

we have

$$
\begin{aligned}
& \left(\frac{\tau\left(\bar{\chi}_{1} \chi_{2}\right)}{\tau\left(\bar{\chi}_{1}\right)}\right)^{2} \\
= & \frac{1}{p^{2}} \cdot\left(\overline{\tau\left(\bar{\chi}_{1}\right)} \cdot \tau\left(\bar{\chi}_{1} \chi_{2}\right)\right)^{2}
\end{aligned}
$$




$$
\begin{aligned}
& =\frac{1}{p^{2}}\left(\sum_{a=1}^{p-1} \chi_{1}(a) e\left(\frac{-a}{p}\right) \sum_{b=1}^{p-1} \bar{\chi}_{1}(b) \chi_{2}(b) e\left(\frac{b}{p}\right)\right)^{2} \\
& =\frac{1}{p^{2}}\left(\sum_{a=1}^{p-1} \chi_{1}(a) \sum_{b=1}^{p-1} \chi_{2}(b) e\left(\frac{b-a b}{p}\right)\right)^{2} \\
& =\frac{1}{p^{2}}\left(\sum_{a=1}^{p-1} \chi_{1}(a) \chi_{2}(1-a) \sum_{b=1}^{p-1} \chi_{2}(b) e\left(\frac{b}{p}\right)\right)^{2} \\
& =\frac{(-1)^{\frac{p-1}{2}}}{p} \cdot\left(\sum_{a=1}^{p-1} \chi_{1}(a) \chi_{2}(1-a)\right)^{2} \\
& =\frac{(-1)^{\frac{p-1}{2}}}{p} \cdot \sum_{a=1}^{p-1} \chi_{1}(a)\left(\frac{1-a}{p}\right) \sum_{b=1}^{p-1} \chi_{1}(b)\left(\frac{1-b}{p}\right) \\
& =\frac{(-1)^{\frac{p-1}{2}}}{p} \cdot \sum_{a=1}^{p-1} \chi_{1}(a) \sum_{b=1}^{p-1}\left(\frac{1-a \bar{b}}{p}\right)\left(\frac{1-b}{p}\right) \\
& =\frac{(-1)^{\frac{p-1}{2}}}{p} \cdot \sum_{a=1}^{p-1} \chi_{1}(a) \sum_{b=1}^{p-1}\left(\frac{b(b-a)}{p}\right)\left(\frac{1-b}{p}\right) \\
& =\frac{(-1)^{\frac{p-1}{2}}}{p} \cdot \sum_{a=1}^{p-1} \chi_{1}(a) \sum_{b=1}^{p-1}\left(\frac{b(b-1)(1-b a)}{p}\right) .
\end{aligned}
$$

Similarly, we also have

(4)

$$
\begin{aligned}
& \overline{\left(\frac{\tau\left(\bar{\chi}_{1} \chi_{2}\right)}{\tau\left(\bar{\chi}_{1}\right)}\right)^{2}} \\
= & \frac{(-1)^{\frac{p-1}{2}}}{p} \cdot \sum_{a=1}^{p-1} \bar{\chi}_{1}(a) \sum_{b=1}^{p-1}\left(\frac{b(b-1)(1-b a)}{p}\right) \\
= & \frac{(-1)^{\frac{p-1}{2}}}{p} \cdot \sum_{a=1}^{p-1} \chi_{1}(a) \sum_{b=1}^{p-1}\left(\frac{b(b-1)(1-b \bar{a})}{p}\right) \\
= & \frac{(-1)^{\frac{p-1}{2}}}{p} \cdot \sum_{a=1}^{p-1} \chi_{1}(a) \sum_{b=1}^{p-1}\left(\frac{a b(b-1)(a-b)}{p}\right) \\
= & \frac{(-1)^{\frac{p-1}{2}}}{p} \cdot \sum_{a=1}^{p-1} \chi_{1}(a) \sum_{b=1}^{p-1}\left(\frac{a b a(b a-1)(a-b a)}{p}\right) \\
= & \frac{(-1)^{\frac{p-1}{2}}}{p} \cdot \sum_{a=1}^{p-1} \chi_{1}(a) \sum_{b=1}^{p-1}\left(\frac{a}{p}\right)\left(\frac{b(b-1)(1-b a)}{p}\right) .
\end{aligned}
$$


Combining (3), (4) and note that $\chi=\chi_{1}^{2}$ we may immediately deduce

$$
\begin{aligned}
& \left(\frac{\tau\left(\bar{\chi}_{1} \chi_{2}\right)}{\tau\left(\bar{\chi}_{1}\right)}\right)^{2}+\overline{\left(\frac{\tau\left(\bar{\chi}_{1} \chi_{2}\right)}{\tau\left(\bar{\chi}_{1}\right)}\right)^{2}} \\
= & \frac{(-1)^{\frac{p-1}{2}}}{p} \cdot \sum_{a=1}^{p-1} \chi_{1}(a)\left[1+\left(\frac{a}{p}\right)\right] \sum_{b=1}^{p-1}\left(\frac{b(b-1)(1-b a)}{p}\right) \\
= & \frac{(-1)^{\frac{p-1}{2}}}{p} \cdot \sum_{a=1}^{p-1} \chi_{1}(a)\left[1+\left(\frac{a}{p}\right)\right] \sum_{b=1}^{p-1}\left(\frac{b(b-1)(1-b a)}{p}\right) \\
= & \frac{(-1)^{\frac{p-1}{2}}}{p} \cdot \sum_{a=1}^{p-1} \chi_{1}\left(a^{2}\right) \sum_{b=1}^{p-1}\left(\frac{b(b-1)\left(1-b a^{2}\right)}{p}\right) \\
= & \frac{(-1)^{\frac{p-1}{2}}}{p} \cdot \sum_{a=1}^{p-1} \chi(a) \sum_{b=1}^{p-1}\left(\frac{b(b-1)\left(1-b a^{2}\right)}{p}\right) .
\end{aligned}
$$

This proves Lemma 2 .

Lemma 3. Let $p$ be an odd prime. Then for any Dirichlet character $\lambda \bmod p$, we have the identities

$$
\sum_{m=1}^{p-1}\left(\frac{m}{p}\right) \cdot\left|\sum_{a=1}^{p-1} \lambda(a) e\left(\frac{m a+\bar{a}}{p}\right)\right|^{2}= \begin{cases}-p, & \text { if } \lambda \neq\left(\frac{*}{p}\right) \\ p(p-2), & \text { if } \lambda=\left(\frac{*}{p}\right) .\end{cases}
$$

Proof. It is clear that from the properties of the reduced residue system mod $p$ and Gauss sums we have

$$
\begin{aligned}
& \sum_{m=1}^{p-1}\left(\frac{m}{p}\right) \cdot\left|\sum_{a=1}^{p-1} \lambda(a) e\left(\frac{m a+\bar{a}}{p}\right)\right|^{2} \\
= & \sum_{m=1}^{p-1}\left(\frac{m}{p}\right) \sum_{a=1}^{p-1} \sum_{b=1}^{p-1} \lambda(a \bar{b}) e\left(\frac{m(a-b)+\bar{a}-\bar{b}}{p}\right) \\
= & \sum_{m=1}^{p-1}\left(\frac{m}{p}\right) \sum_{a=1}^{p-1} \lambda(a) \sum_{b=1}^{p-1} e\left(\frac{m b(a-1)+\bar{b}(\bar{a}-1)}{p}\right) \\
= & \sum_{a=1}^{p-1} \lambda(a) \sum_{b=1}^{p-1} \sum_{m=1}^{p-1}\left(\frac{m}{p}\right) e\left(\frac{m b(a-1)+\bar{b}(\bar{a}-1)}{p}\right) \\
= & \left(\sum_{m=1}^{p-1}\left(\frac{m}{p}\right) e\left(\frac{m}{p}\right)\right) \sum_{a=1}^{p-1} \lambda(a) \sum_{b=1}^{p-1}\left(\frac{b(a-1)}{p}\right) e\left(\frac{\bar{b}(\bar{a}-1)}{p}\right) \\
= & \left(\sum_{m=1}^{p-1}\left(\frac{m}{p}\right) e\left(\frac{m}{p}\right)\right) \sum_{a=1}^{p-1} \lambda(a) \sum_{b=1}^{p-1}\left(\frac{b(a-1)}{p}\right) e\left(\frac{b(\bar{a}-1)}{p}\right)
\end{aligned}
$$




$$
\begin{aligned}
& =\left(\sum_{m=1}^{p-1}\left(\frac{m}{p}\right) e\left(\frac{m}{p}\right)\right)^{2} \sum_{a=1}^{p-1} \lambda(a)\left(\frac{(a-1)(\bar{a}-1)}{p}\right) \\
& =p \cdot \sum_{a=1}^{p-1} \lambda(a)\left(\frac{a(a-1)^{2}}{p}\right)=p \cdot \sum_{a=2}^{p-1} \lambda(a)\left(\frac{a}{p}\right) \\
& =p \cdot\left(\sum_{a=1}^{p-1} \lambda(a)\left(\frac{a}{p}\right)-1\right)= \begin{cases}-p, & \text { if } \lambda \neq\left(\frac{*}{p}\right) ; \\
p(p-2), & \text { if } \lambda=\left(\frac{*}{p}\right) .\end{cases}
\end{aligned}
$$

This proves Lemma 3 .

\section{Proof of Theorem}

In this section, we shall complete the proof of our theorem. In fact from Lemma 1, Lemma 2 and the properties of the Gauss sums we have

$$
\begin{aligned}
& \left|\sum_{a=1}^{p-1} \chi(m a+\bar{a})\right|^{2} \\
= & p \cdot\left|1+\left(\frac{m}{p}\right)\left(\frac{\tau\left(\bar{\chi}_{1} \chi_{2}\right)}{\tau\left(\bar{\chi}_{1}\right)}\right)^{2}\right|^{2} \\
= & p \cdot\left(2+\left(\frac{m}{p}\right)\left(\left(\frac{\tau\left(\bar{\chi}_{1} \chi_{2}\right)}{\tau\left(\bar{\chi}_{1}\right)}\right)^{2}+\overline{\left(\frac{\tau\left(\bar{\chi}_{1} \chi_{2}\right)}{\tau\left(\bar{\chi}_{1}\right)}\right)^{2}}\right)\right) \\
= & 2 p+(-1)^{\frac{p-1}{2}}\left(\frac{m}{p}\right) \sum_{a=1}^{p-1} \chi(a) \sum_{b=1}^{p-1}\left(\frac{b(b-1)\left(1-b a^{2}\right)}{p}\right) .
\end{aligned}
$$

Note that the identity

(6)

$$
\begin{aligned}
& \sum_{m=1}^{p-1}\left|\sum_{b=1}^{p-1} \lambda(b) e\left(\frac{m b+\bar{b}}{p}\right)\right|^{2} \\
= & \sum_{m=1}^{p}\left|\sum_{b=1}^{p-1} \lambda(b) e\left(\frac{m b+\bar{b}}{p}\right)\right|^{2}-\left|\sum_{b=1}^{p-1} \lambda(b) e\left(\frac{\bar{a}}{p}\right)\right|^{2} \\
= & \begin{cases}p^{2}-p-1, & \text { if } \lambda \text { is the principal character mod } p ; \\
p(p-2), & \text { if } \lambda \text { is not the principal character mod } p .\end{cases}
\end{aligned}
$$

From (5), (6) and Lemma 3 we have

(7)

$$
\sum_{m=1}^{p-1}\left|\sum_{b=1}^{p-1} \lambda(b) e\left(\frac{m b+\bar{b}}{p}\right)\right|^{2} \cdot\left|\sum_{a=1}^{p-1} \chi(m a+\bar{a})\right|^{2}
$$




$$
\begin{aligned}
= & (-1)^{\frac{p-1}{2}} \sum_{m=1}^{p-1}\left(\frac{m}{p}\right)\left|\sum_{b=1}^{p-1} \lambda(b) e\left(\frac{m b+\bar{b}}{p}\right)\right|^{2} \sum_{a=1}^{p-1} \chi(a) \sum_{b=1}^{p-1}\left(\frac{b(b-1)\left(1-b a^{2}\right)}{p}\right) \\
& +2 p \cdot \sum_{m=1}^{p-1}\left|\sum_{b=1}^{p-1} \lambda(b) e\left(\frac{m b+\bar{b}}{p}\right)\right|^{2} \\
= & 2 p^{3}+O\left(p^{2}\right)-(-1)^{\frac{p-1}{2}} \cdot p \cdot \sum_{a=1}^{p-1} \chi(a) \sum_{b=1}^{p-1}\left(\frac{b(b-1)\left(1-b a^{2}\right)}{p}\right) .
\end{aligned}
$$

From (5), Lemma 1 and the properties of the Gauss sums we have the estimate

$$
\left|\sum_{a=1}^{p-1} \chi(a) \sum_{b=1}^{p-1}\left(\frac{b(b-1)\left(1-b a^{2}\right)}{p}\right)\right|=\left.|| \sum_{a=1}^{p-1} \chi(m a+\bar{a})\right|^{2}-2 p \mid \ll p .
$$

Combining (7) and (8) we may immediately deduce the asymptotic formula

$$
\sum_{m=1}^{p-1}\left|\sum_{b=1}^{p-1} \lambda(b) e\left(\frac{m b+\bar{b}}{p}\right)\right|^{2} \cdot\left|\sum_{a=1}^{p-1} \chi(m a+\bar{a})\right|^{2}=2 p^{3}+O\left(p^{2}\right) .
$$

This completes the proof of our theorem.

Acknowledgment. The author would like to thank the referee for his very helpful and detailed comments, which have significantly improved the presentation of this paper.

\section{References}

[1] Tom M. Apostol, Introduction to Analytic Number Theory, Springer-Verlag, New York, 1976.

[2] D. A. Burgess, On character sums and primitive roots, Proc. London Math. Soc. 12 (1962), 179-192.

[3] $\longrightarrow$ On Dirichlet characters of polynomials, Proc. London Math. Soc. 13 (1963), $537-548$.

[4] A. Granville and K. Soundararajan, Large character sums: Pretentious characters and the Pólya-Vinogradov theorem, J. Amer. Math. Soc. 20 (2007), no. 2, 357-384.

[5] W. Zhang and W. Yao, A note on the Dirichlet characters of polynomials, Acta Arith. 115 (2004), no. 3, 225-229.

[6] W. Zhang and Y. Yi, On Dirichlet characters of polynomials, Bull. London Math. Soc. 34 (2002), no. 4, 469-473.

Department of Mathematics

NorthWESt University

XI'An, ShaAnxi, P. R. China

E-mail address: wangjingzhe729@126.com 